\section{Push-button Control}

from our Molecular Biology Correspondent ENZYMOLOGY is clearly getting harder every day. Time was when one enzyme was considered to do one job, and the only legitimate objective of the enzymologist was to find out how. An increasing number of enzymes are now coming to light which can be switched on, off, or even into other modes, in response to triggering ligands. A very advanced model is that described by Meister and his colleagues (Trotta $e t$ al., Proc. US Nat. Acad. Sci., 68, 2599; 1971). This is a carbamyl phosphate synthetase which, like other members of the family of glutamine amidotransferases, utilizes the amide group of glutamine for various synthetic functions. It is a characteristic of this group of enzymes that they are also able to make do with ammonia, though this is turned over with lower efficiency.

The Escherichia coli carbamyl phosphate synthetase puts together its endproduct from glutamine, bicarbonate and ATP and intermediate steps in this process can also be isolated. The enzyme is controlled by several positive and negative feedback ligands, which are formed at the ends of the synthetic pathways in which it is involved. Trotta et al. have now demonstrated that the molecule consists of two subunits, estimated by detergent-gel electrophoresis to have molecular weights of 42,000 and 130,000 . These chains could be dissociated, separated, and then recombined to give a substantially native product.

Examination of the activities of the separated subunits showed the following: neither chain alone was capable of the synthesis of carbamyl phosphate from glutamine, nor of the partial reaction of glutamylhydroxamate hydrolysis in the presence of ATP and bicarbonate. The large subunit, on the other hand, was active in carbamyl phosphate synthesis from ammonia rather than glutamine, and also in another partial reaction-the hydrolysis of ATP in the presence of bicarbonate. Both chains individually retained the glutaminase function. The various activators and inhibitors still exert their control over the activity of the large subunit, which therefore evidently contains the relevant binding sites. The function of the small subunit is surmised to be the binding of glutamine to a site at which it can be attacked by a nucleophile, which by an affinity label experiment with a glutamine analogue is inferred to be a thiol group. Trotta et al. suggest from a comparison of related enzymes in other species that the small subunit may represent an evolutionary refinement, in which ammonia was supplanted as the main nitrogen source by glutamine. A test of this hypothesis would be a structural comparison of glutamine-binding chains from the related enzymes of other species.

A system with great biochemical appeal just now are the cyclic AMP dependent protein kinases, which have been identified in a number of tissues. These seem always to consist of two subunits, one catalytic and one regulatory, and the word now is that the cyclic AMP operates by binding to the latter. In Krebs's laboratory a direct demonstration of this scheme in the rabbit skeletal muscle enzyme has now been achieved (Brostrom et al., ibid., 2444). Two kinases, differing somewhat in molecular weight, are present in the tissue. Each consists of a regulatory and a catalytic subunit, and it is only the former which differ in size. Mole for mole the two proteins are identical in AMP binding capacity. When cyclic AMP is added to the enzyme, the subunits dissociate, and can be separated chromatographically, the ligand being then found only on the regulatory chain. Short of denaturation, the bound cyclic AMP could not be removed from the protein, except by addition of an excess of the catalytic subunit, when it was forthwith displaced.

It seems then that there the system functions by a disproportionation mechanism: cyclic AMP will bind to the regulatory subunit, and thereby displace it from its partner, or the catalytic subunit may bind to the regulatory chain, with displacement of the cyclic AMP. In practical terms the liganded regulatory subunit when added to the intact kinase increases the cyclic AMP concentration required for a given level of kinase activity. If some agency (insulin being an interesting candidate) were to cause a diminution in cyclic AMP concentration, the equilibrium between the kinase subunits would shift in favour of association, and the activity would become more sensitive to the cyclic AMP that remains. Brostrom et al. suggest that the complex with the regulatory subunit may be the form in which the cyclic AMP is kept in readiness in the cell. The next step will be to measure the concentrations of the two subunits actually present.

The protein kinases in liver have been studied by Chen and Walsh (Bio chemistry, 10, 3614; 1971). They are three in number, two, of apparently identical molecular weight, being cyclicAMP-controlled, and a smaller one, which is not. The preponderant heavy component is heterogeneous, in that catalytic subunit can be resolved into two components. The smaller kinase is evidently an isolated catalytic chain, but is not identical with the other catalytic subunits. On the other hand, it will combine functionally with isolated regulatory subunits. Chen and Walsh note that there are many synthetic functions in the liver which might be expected to be regulated by cyclic AMP, and might therefore call for the presence of different specialized protein kinases.

\title{
Peptide Map of Chimpanzee Haemoglobin
}

THE haemoglobins of higher primates are very like those of man. In the chimpanzee it is thought that all the three major polypeptide chains $(\alpha, \beta$ and $\gamma$ chains, the last of which is the foetal analogue of the $\beta$ chain) have identical sequences to the corresponding human molecules. Moreover, the $\gamma$-chain exists in two forms, one with glycine, the other with alanine at position 136 , in monkeys, apes and man. Again the chimpanzee possesses the minor adult component, $\mathrm{A}_{2}$, which has the structure $\alpha_{2}, \delta_{2}$, just as in humans, and it is the $\delta$-chains which are the subject of a sequence study by De Jong, reported in next Wednesday's issue of Nature New Biology.

The tryptic peptide map of chimpanzee $\delta$-chains is identical with that of human $\delta$-chains, except for one peptide. The substitution turns out to be val-126 for the met-126 found in man. It is highly probable that there are no other differences between the sequences. The replacement corresponds to a point mutation (GUG $\rightarrow$ AUG) which would have occurred after evolutionary divergence, valine occupying the corresponding position in the molecule in all known primate $\alpha, \beta$ and $\gamma$-chains, and also in the $\delta$-chains of some New World monkeys. (Old World monkeys seem to have no haemoglobin $\mathrm{A}_{2}$.)

Calculations have shown that the rate of evolutionary mutation in mammalian haemoglobins is one substitution for 100 residues every $10^{7}$ years. With 725 residues in the normal haemoglobin chains $(\alpha, \beta$, two types of $\gamma$ and the $\delta$ ) altogether, the statistically predicted extent of mutation would be one residue between all of them every 1.4 million years. The divergence time for hominids is variously reckoned to be between 4 and 14 million years ago, and De Jong argues that the absence of any replacements whatever since divergence is good evidence in favour of the shorter time. 